Ann. Zootech., I970, 19 (3), 333-346.

\title{
INFLUENCE DES RESTRICTIONS ALIMENTAIRES INTERVENANT DÈS LA NAISSANCE SUR LES PERFORMANCES DE PONTE DE DEUX SOUCHES DE POULETTES
}

\author{
B. LECLERCQ, J. SIMON, J.-C. BLUM et C. CALE'T \\ Station de Recherches avicoles, \\ Centre de Recherches de Tours, 37 - Nouzilly \\ Institut national de la Recherche agronomique
}

\section{RÉSUUMÉ}

Des restrictions d'une extrême sévérité sont imposées à des poussins femelles de souche lourde (type White Rock) et légère (type Leghorn) depuis la naissance jusqu'à 20 semaines. Les régimes isoénergétiques (2 850 cal. mét. $/ \mathrm{kg}$ ) se distinguent seulement par leur teneur et leur composition en protéines. Mille deux cents poussins de chaque souche sont répartis en 4 lots. Le lot de référence (T) consomme ad libitum un aliment équilibré assurant une croissance rapide : 2I p. roo de protéines entre o et 6 semaines; r 8 p. Ioo de 6 à I 2 semaines, I 5 p. Ioo ensuite. Le lot S reçoit les mêmes régimes mais est alimenté périodiquement (Skip-a-day) : 6 jours sur 7 jusqu'à I2 semaines, 5 jours sur 7 entre 12 et 20 semaines. Les protéines du lot P (Io p. Ioo) sont fournies presque exclusivement par des céréales jusqu'à 3 semaines ; au-delà le taux protidique du régime est relevé à I 3 p. Ioo au moyen de farines animales qui assurent un meilleur équilibre des acides aminés indispensables. Enfin, le lot $\mathrm{A}$ reçoit alternativement le régime céréalier (1o p. Ioo de protéines) pendant $\mathrm{I}_{4}$ jours puis le régime à $2 \mathrm{I} \mathrm{p}$. Ioo de protéines pendant 7 jours. Ce rythme (I4 jours-7 jours) est maintenu pendant toute la période de croissance. Les sujets de chaque lot disposent de l'aliment à volonté chaque fois qu'ils y ont accès. A partir de l'âge de 20 semaines et pour toute la période de ponte, tous les animaux d'une même souche disposent ad libitum d'un régime identique qui satisfait leurs besoins.

L'influence des restrictions imposées pendant le jeune âge est liée à la réduction quantitative de la consommation. Dans le lot $\mathrm{S}$ les animaux compensent en partie l'effet des jeûnes périodiques en surconsommant pendant les phases de réplétion : le développement (croissance, maturité sexuelle) est modérément retardé. Dans les lots $\mathrm{P}$ et A les modifications sont plus profondes : croissance très ralentie, maturité sexuelle retardée d'une vingtaine de jours. Il existe une interaction très significative entre la souche et le mode de restriction. La maturité sexuelle la plus retardée se manifeste chez les Leghorn du lot A alors qu'elle est observée chez les White Rock du lot P.

Pour l'ensemble des deux souches les performances de ponte ne sont pas profondément modifiées par les restrictions imposées durant le jeune âge. Ces restrictions protidiques, très intenses dans les lots $\mathrm{P}$ et $\mathrm{A}$, provoquent une diminution légère mais significative des performances ultérieures de ponte chez les Leghorn; dans la souche White Rock, au contraire, elles ont toujours un effet favorable. Dans tous les cas, elles n'affectent pas le poids moyen de l'œuf. On peut donc envisager de rationner les animaux de souche lourde dès l'éclosion. Ceci peut être réalisé par l'utilisation d'un seul régime de taux protidique moyen. Cette déficience ralentira la croissance pendant les premières semaines d'élevage ; le retard étant ensuite maintenu par un rationnement quantitatif. 


\section{INTRODUCTION}

Le rationnement des potlettes pendant la période de croissance est devenu d'usage courant. Il présente plusieurs avantages : économie sur la quantité d'aliment consommé, animaux plus maigres, retard de la maturité sexuelle et de ce fait augmentation du poids moyen des œufs pondus en début de ponte. Variables dans leurs modalités d'application, les restrictions alimentaires reposent toujours sur l'un des deux principes suivants : distribution d'un aliment complet et équilibré en quantité limitée, distribution d'un aliment déficient (protéines, acides aminés...). Il s'ensuit un retard de croissance et une réduction de la quantité d'aliment consommé. Les programmes de rationnement qui découlent de ces méthodes sont souvent analogues. Mais on peut distinguer ceux (très nombreux et variés) qui ne font commencer le traitement qu'à partir de l'âge de 6 ou 8 semaines et ceux, exceptionnels, qui sont mis en œuvre dès l'éclosion (SINGSEN, I965). Dans le premier cas on assiste à une croissance normale, voire accélérée, au cours des premières semaines que l'on s'évertue ensuite de ralentir. Ce programme ne représente pas forcément la meilleure solution et il paraît nécessaire de préciser l'âge à partir duquel il est bon de restreindre les animaux. Par ailleurs il est rare que les effets de divers types de restriction soient comparés sur des souches différentes. Or, certains résultats contradictoires rencontrés dans la bibliographie pourraient être imputés à 1'utilisation d'animaux dissemblables sur le plan génétique. Nous avons voulu intervenir sur le montant des éléments nutritifs ingérés par 1'animal au moyen de diverses modalités de rationnement. Parmi celles-ci, nous nous sommes adressés à l'alimentation alternée dont l'intérêt avait été mis à profit par deux d'entre nous dans le cas du poulet (Simon, BLum et JACQUOT, I968). En outre, nous avons essayé de déterminer dans quelle mesure le jeune âge et la souche sont importants.

\section{MATÉRIEL, E'T MÉTHODES}

\section{Animaux}

Issus de croisements commerciaux, ils sont du type Leghorn, souche légère destinée à la production des oeufs de consommation, ou du type White Rock, animaux plus lourds destinés à la production du poulet de chair. Pendant la croissance (o à 20 semaines), les 2400 poulettes, I 200 de chaque souche, sont élevées sur litière ; elles disposent d'une période journalière d'éclairement de $\mathrm{I}_{4}$ heures. A l'intérieur de chaque souche, on constitue 4 lots de 300 poulettes ; à l'âge de I2 semaines quelques-uns des animaux sont éliminés au hasard afin de décomposer chaque lot en 3 répétitions de 7o sujets chacune. Une nouvelle réduction des effectifs intervenant à l'âge de 20 semaines laisse subsister 50 individus. Chacune des 24 répétitions $(3 \times 4$ lots $\times 2$ souches) occupe une case de ponte de $I_{5} \mathrm{~m}^{2}$ dans un poulailler sur litière. Les cases sont munies de nidstrappes; des abreuvoirs automatiques fournissent l'eau de boisson à profusion. L'éclairement journalier est de 18 heures pendant toute la durée de la ponte.

\section{Régimes et modes d'alimentation selon les lots}

La composition des régimes figure dans le tableau I. On doit distinguer deux périodes dans la vie de l'animal. 
a) Période d'élevage (o à 20 semaines).

Les 5 régimes utilisés sont isoénergétiques, également supplémentés en vitamines et minéraux. Nous les désignerons par leur teneur en protéines qui varie d'un régime à l'autre : 2I p. Ioo, I 8 p. Ioo, I5 p. Ioo, I3 p. roo, Io p. Ioo. Dans les quatre premiers régimes, l'équilibre entre

\section{TABLEAU I}

Composition des régimes utilisés pour la croissance

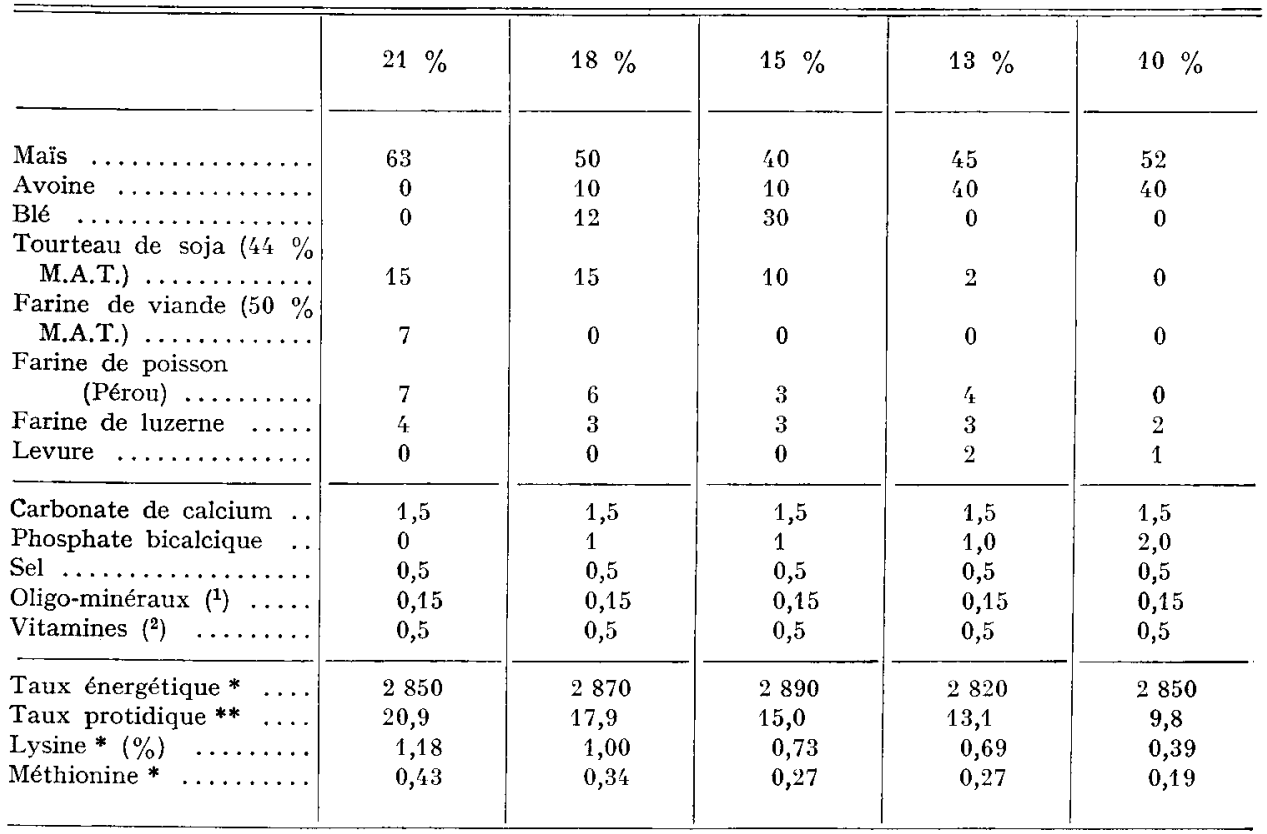

(1) Composition du mélange minéral: (par $\mathrm{kg}$ de mélange) sulfate de cuivre : $7 \mathrm{~g}$, sulfate de cobalt : $1 \mathrm{~g}$, molybdate de sodium : $0,5 \mathrm{~g}$, sulfate de zinc : $150 \mathrm{~g}$, iodure de potassium : $1,5 \mathrm{~g}$, carbonate de calcium : $335 \mathrm{~g}$, sulfate de manganèse : $200 \mathrm{~g}$, oxyde de magnésie : $100 \mathrm{~g}$, carbonate de magnésie : $80 \mathrm{~g}$, sulfate de fer : $75 \mathrm{~g}$

(2) Composition du mélange vitaminique : (par $\mathrm{kg}$ de mélange) vitamine A : $1600000 \mathrm{UI}$, vitamine $\mathrm{D}_{\mathrm{a}}: 200000 \mathrm{UI}$, riboflavine $: 0,6 \mathrm{~g}$, pantothénate de calcium : 1,'1 $\mathrm{g}$, nicotinamide : $2 \mathrm{~g}$, vitamine $\mathrm{E}:$ $8 \mathrm{~g}$, choline : $80 \mathrm{~g}$, B.H.T. $: 20 \mathrm{~g}$.

* Calculé; ** Mesuré.

acides aminés indispensables est satisfaisant, par contre, l'aliment ro p. Ioo composé presque exclusivement de céréales est très déficient, il n'apporte que des protéines médiocres en faible quantité. Les poulettes White Rock et Leghorn sont alimentées suivant les mêmes protocoles. Dans chaque souche il existe 4 lots :

- Le lot $T$ (Témoin) dispose ad libitum d'une alimentation assurant une croissance rapide : régime 2 I p. Ioo de o à 6 semaines, I 8 p. Ioo de 6 à I 2 semaines, I 5 p. roo ensuite.

- Le lot S (Skip-a-day) consomme les mêmes régimes que le lot Témoin, mais il n'est alimenté que périodiquement : 6 jours sur 7 de o à 12 semaines ; 5 jours sur 7 de 12 à 20 semaines, les animaux jeûnant le jeudi et le dimanche.

- Le lot P (restreint en protéines) est nourri ad libitum avec le régime ro p. Ioo jusqu'a l'âge de 3 semaines et avec le régime 13 p. roo par la suite.

- Le lot A (alterné) reçoit alternativement le régime io p. roo pendant $\mathrm{r}_{4}$ jours, puis le régime 2 I p. Ioo pendant 7 jours. Ce rythme, deux semaines d'alimentation déficiente en protéines pour une semaine avec régime riche, est maintenu pendant toute la période de croissance. 
Selon les lots, les animaux ont ou n'ont pas d'aliment à leur disposition à plein-temps, mais lorsqu'ils en disposent, ils peuvent le consommer sans contrainte. On a veillé à ce que le nombre des mangeoires soit suffisant pour que tous les sujets puissent y avoir accès en même temps.

b) Période de ponte.

A partir de 20 semaines d'âge tous les animaux sont alimentés ad libitum. Il n'existe que deux régimes. L'un est distribué à toutes les poulettes Leghorn, l'autre à toutes les poulettes White Rock, quel que soit leur lot d'origine. Les régimes ont une composition adaptée aux besoins de chaque souche (cf. tabl. 2). D'après les normes habituellement admises ils doivent permettre une bonne ponte tout en limitant l'engraissement dans la souche White Rock qui a tendance à surconsommer.

TABLEAU 2

Composition des régimes utilisés pour la ponte

\begin{tabular}{|c|c|c|}
\hline & Leghorn & White Rock \\
\hline$\ldots \ldots \ldots \ldots$ & 1 & 0 \\
\hline 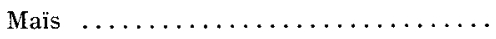 & 47 & 35 \\
\hline Avoine $\ldots \ldots \ldots \ldots \ldots \ldots \ldots \ldots$ & 23 & 25 \\
\hline Remoulage $\ldots \ldots \ldots \ldots \ldots \ldots \ldots$ & 0 & 19 \\
\hline Tourteau de soja (44 $\%$ de M.A.T.) ... & 10 & 0 \\
\hline Tourteau de tournesol $\ldots . . \ldots \ldots \ldots$ & 0 & 3 \\
\hline Farine de poisson (Pérou) $\ldots \ldots \ldots \ldots$ & 0 & 2 \\
\hline Farine de viande $\left({ }^{\prime} 0 \%\right.$ M.A.T. $) \ldots \ldots$ & 9 & 2 \\
\hline Farine de luzerne $\ldots \ldots \ldots \ldots \ldots$ & 4 & 8 \\
\hline Carbonate de calcium $\ldots \ldots \ldots \ldots$ & 4 & 4 \\
\hline Phosphate bicalcique..$\ldots \ldots \ldots \ldots$ & 1,35 & 1,35 \\
\hline Oligo-minéraux $(\mathbf{1}) \ldots \ldots \ldots \ldots \ldots$ & 0,15 & 0,15 \\
\hline Mélange vitaminique $\left(^{1}\right) \ldots \ldots \ldots \ldots$ & 0,5 & 0,5 \\
\hline
\end{tabular}

(1) Voir tableau 1

\section{Mesures effectuées}

Les animaux sont pesés individuellement aux âges de $6,12,20$ et 60 semaines (fin de la période de ponte). La ponte est contrôlée individuellement 5 jours par semaine et les résultats sont groupés par mensualité. Le poids moyen de l'œuf est déterminé une fois par mois en pesant les ceufs pondus par chaque poule pendant 5 jours consécutifs. La consommation alimentaire est également enregistrée par mensualité pour chaque répétition d'animaux (3 répétitions par lot).

\section{Analyse statistique}

Pour chaque mesure on dégage l'effet "souche \#, l'effet "régime " de la période d'élevage et l'interaction entre ces deux facteurs. L'analyse de variance de ce dispositif factoriel est réalisée grâce à la méthode des "Fitting constants " (SNEDECOR, 1956), méthode qui est utilisée parce que le nombre d'observations est différent dans chaque traitement.

\section{RÉSULTATS}

\section{I. - Période de croissance}

\section{Développement pondéral.}

Le tableau 3 indique le poids moyen des animaux et le résultat des analyses statistiques aux âges de 6 , I2 et 20 semaines. Les restrictions alimentaires retardent 
RESTRICTION PREACOCE, DE LA POULETTE

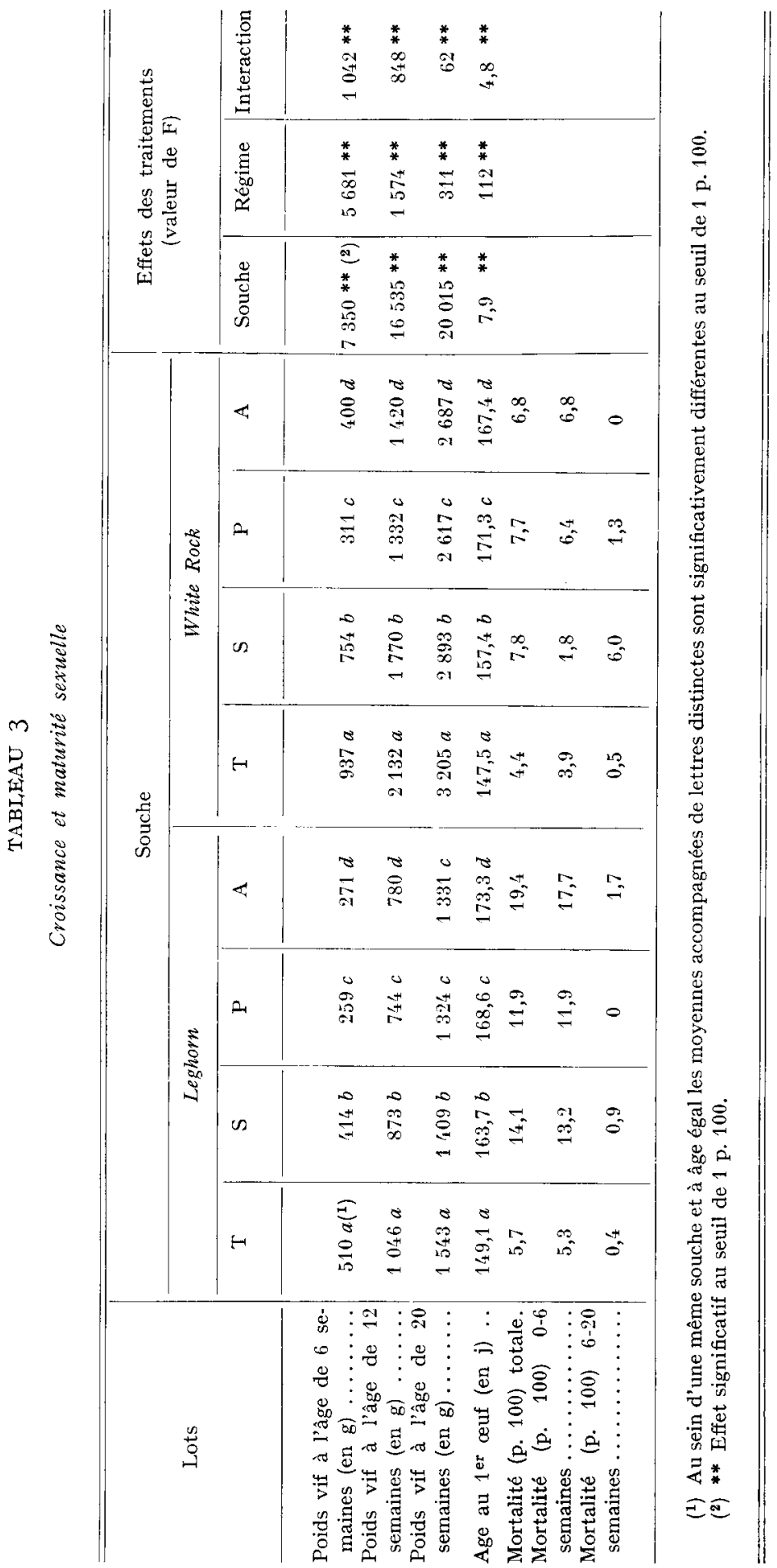


la croissance. Lorsqu'on classe les lots en fonction du poids vif, il apparait une hiérarchie qui est la même pour les deux souches, des animaux les plus lourds aux plus légers on note le lot $\mathrm{T}$, le lot $\mathrm{S}$, le lot $\mathrm{A}$, puis le lot $\mathrm{P}$. Alors que les deux souches subissent le même retard de croissance avec la distribution en skip-a-day (lot $\mathrm{S}$ ) elles réagissent plus profondément mais aussi différemment dans les deux autres lots. La souche lourde est particulièrement touchée : en pourcentage du lot $\mathrm{T}$ les gains de poids à l'âge de 6 semaines sont respectivement pour $\mathrm{P}$ et $\mathrm{A}$ de 33 et 43 chez les White Rock et de 5 I et $5^{2}$ chez les Leghorn.

\section{Mortalité.}

Les résultats figurent également dans le tableaı 2. La mortalité est plus élevée dans les lots restreints. Dans la souche White Rock, la différence avec les témoins est inférieure à $3,5 \mathrm{p}$. roo entre 0 et 20 semaines. Chez les poulettes Leghorn, la mortalité est importante entre o et 6 semaines; elle est due à des crises de cannibalisme dans les lots restreints tout particulièrement dans le lot A. Le cannibalisme a disparu après adoption d'un éclairage rouge au cours de la $5^{\mathrm{e}}$ semaine.

\section{Consommation d'aliment.}

Le tableau 4 rassemble les consommations globales des animaux pendant les 20 premières semaines de leur développement. Chez les lots $\mathrm{P}$ et $\mathrm{A}$ les économies d'aliment se traduisent bien davantage au niveau azoté qu'énergétique.

\section{TABLEAU 4}

Consommation alimentaire de la poulette entre 0 et 20 semaines

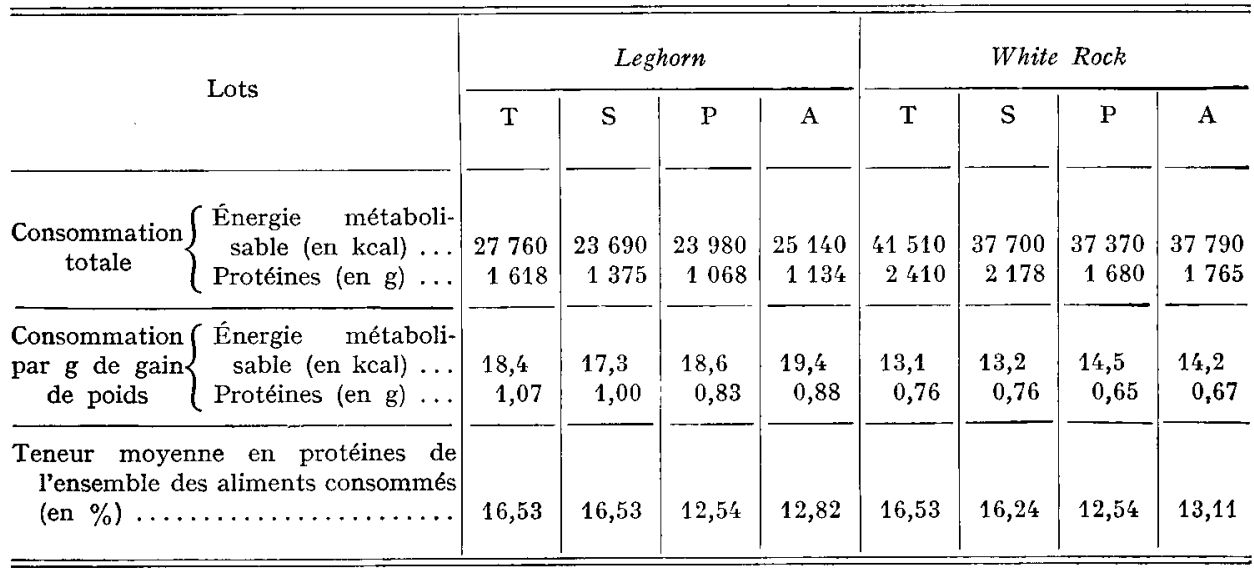

Sous l'effet du jeûne hebdomadaire (lot S) l'efficacité azotée et énergétique est meilleure que chez les témoins dans la souche Leghorn. Il est étonnant qu'il n'en soit pas de même chez les White Rock.

L'alimentation alternée se traduit par une diminution considérable de la part des protéines ingérées dans 1'ensemble des aliments consommés. Cette part est voisine de celle imposée dans le lot P. Il s'ensuit dans ces deux lots une amélioration de 
l'utilisation azotée et une détérioration de l'efficacité énergétique dans les deux souches. A noter toutefois que la souche lourde utilise mieux les aliments que la souche légère.

La figure I illustre l'évolution de la consommation des sujets pendant leur croissance. La réduction des ingestas due au rationnement et surtout à l'abaissement du taux protidique du régime (lot $\mathrm{P}$ ) se fait sentir de o à 6 semaines. Les White Rock
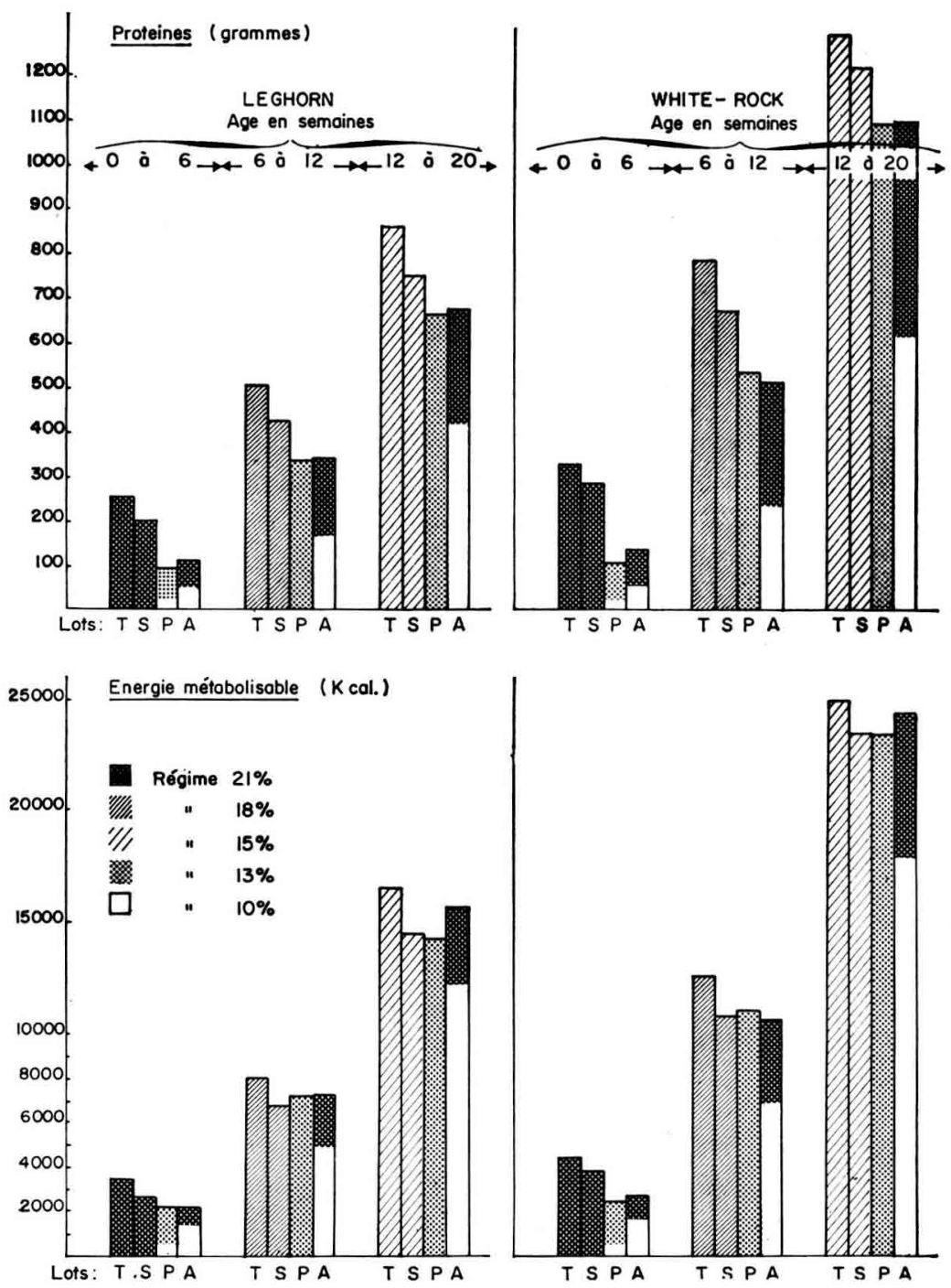

Fig. I. - Quan'ités de protêines et d'énergie consommées au courrs des trois périodes de croissance: $0-6,6-12,12-20$ (semaines)

apprécient encore moins que les Leghorn l'aliment pauvre en protéines (diminutions respectives des quantités ingérées de $4 \mathrm{I}$ et $34 \mathrm{p}$. Ioo par rapport aux témoins). En revanche, elles s'adaptent mieux à cet aliment puisque les différences s'atténuent par 
$1 \mathrm{a}$-suite. Entre 12 et 20 semaines ces valeurs sont en proportion inverse respectivement égales à 6 et $\mathrm{I} 4 \mathrm{p}$. Ioo. Les consommations du lot alterné (A) rendent également compte des besoins azotés plus élevés des White Rock. Disposant une semaine sur trois de l'aliment à 2I p. Ioo de protéines les poulettes White Rock en consomment relativement davantage que les Leghorn. Chez les premières 35,2 p. roo des calories métabolisables proviennent de cet aliment contre $3 \mathrm{I}, 3$ chez les secondes. Ainsi, les White Rock ingèrent plus de protéines totales et beaucoup plus d'acides aminés indispensables que les Leghorn.

\section{Maturité sexuelle.}

\section{II. - Période de reproduction}

L'âge au premier œuf est indiqué dans le tableau 3. La maturité sexuelle est retardée dans tous les lots restreints. Le retard le plus important (environ 24 jours) est observé dans le lot A chez les poulettes Leghorn, dans le lot $\mathrm{P}$ chez les White Rock.

\section{Performances de ponte.}

Elles sont enregistrées pendant 9 mois (du I6 $67^{\mathrm{e}}$ au $440^{\mathrm{e}}$ jour de vie). Les contrôles commencent donc avant ou après le début de la ponte selon les lots.

Les résultats moyens et leur signification statistique sont consignés dans le tableau 5. Le nombre et le poids total d'œufs pondus par poule en fin d'expérience sont influencés par les restrictions subies au cours de la période de croissance. Cette influence est profondément différente selon les souches. Les régimes restreints en protéines $(\mathrm{P})$ et alternés $(\mathrm{A})$ ont un effet significativement défavorable pour la souche légère, le dernier d'entre eux entraîne une réduction des performances de l'ordre de ro p. Ioo. Au contraire pour la souche lourde les trois types de restriction imposées aux animaux ont conduit à une nette augmentation du nombre d'œufs pond us par rapport aux témoins.

La figure 2 montre que dans les 2 souches les lots dont la maturité sexuelle a été la plus retardée par le rationnement $(\mathrm{P}$ et $\mathrm{A})$ pondent à tout moment des oufs plus légers que les animaux témoins de même âge. Étant donné que la date d'entrée en ponte des lots restreints a été retardée, les poules de ces lots pondent moins d'œufs au début et plus d'œufs en fin de la période de ponte ( $167^{\circ}$ au $440^{\mathrm{e}}$ jour de vie) que les témoins. C'est ce qui explique que le poids moyen de l'œuf calculé sur toute la période de ponte n'est guère modifié (tabl. 5).

\section{Développement pondéral et consommation d'aliment.}

Aucun des sujets n'étant rationné pendant la période de ponte, on assiste à un gain pondéral des animaux d'autant plus marqué que les restrictions ont été plus fortes, de même la reprise de poids est beaucoup plus intense chez les White Rock que chez les Leghorn.

La consommation ad libitum a été contrôlée collectivement pour chaque répétition. Comme on ne dispose que de 3 répétitions par lot, il est difficile de mettre en évidence des différences significatives. On constate seulement que les animaux antérieurement restreints tendent à consommer davantage dans la souche Leghorn, tandis que dans la souche White Rock leur consommation est plutôt diminuée. Ce dernier résultat est assez remarquable, étant donné que les animaux ont à la fois une produc- 

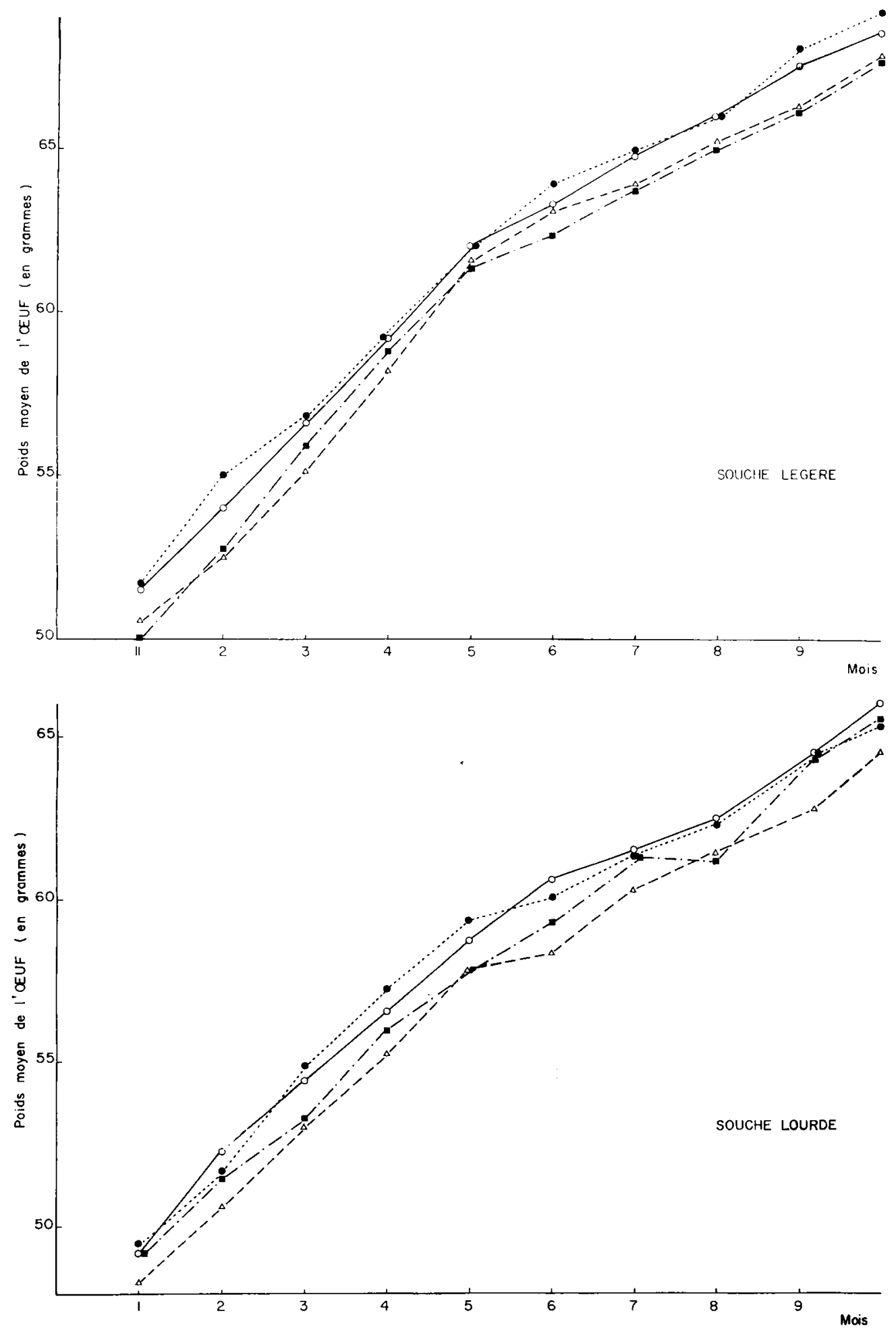

FIG. 2. - Evolution du poids de l'œuf selon les régimes distribués pendant la période de croissance - Témoins - Skip-a-day $\Delta$ TP faible P $\mathbf{P}$ TP alternés A 
B. LECLERCQ, J. SIMON, J.-C. BLUM, C. CALET

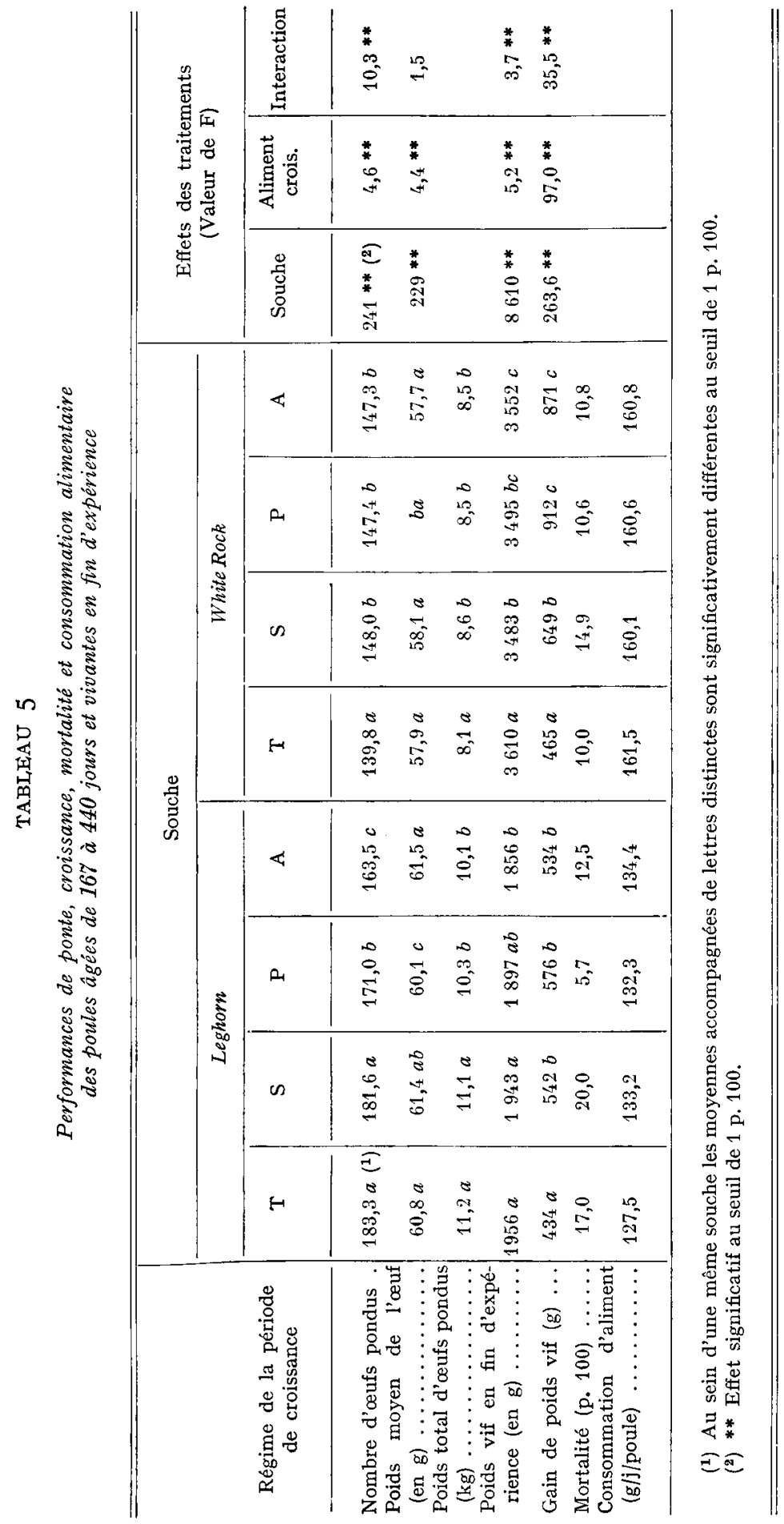


tion d'œufs et un développement pondéral accrus par rapport aux témoins. Autrement dit, l'efficacité alimentaire est augmentée pendant la période de ponte chez les poules de souche lourde antérieurement restreintes.

L'effet des restrictions sur le poids vif n'est pas complètement effacé par les 9 mois d'alimentation ad libitum. En fin d'expérience, les poules Leghorn du lot A et les poules White Rock des 3 lots antérieurement restreints sont très significativement moins lourdes que les animaux témoins.

4. Mortalité.

Elle ne paraît pas influencée par les restrictions alimentaires antérieures.

\section{DISCUSSION}

Les restrictions alimentaires influencent le devenir de tous les animaux. Mais il existe des différences entre souches traduites au niveau de l'analyse statistique par un effet significatif de l'interaction régime-souche. Nous discuterons d'abord des effets comparables dans les deux souches; nous envisagerons ensuite les différences entre souches.

\section{Effets des restrictions alimentaires comparables dans les deux souches}

Les restrictions alimentaires ralentissent la croissance et retardent l'apparition de la maturité sexuelle. Mais les performances de ponte restent satisfaisantes dans tous les cas. Ainsi que l'indiquent HuTT et NESHEIM (I969), la production d'oufs est indépendante de l'âge auquel commence la ponte. Il faut d'ailleurs remarquer que l'âge d'entrée en ponte n'est pas directement lié au développement pondéral à l'âge de 20 semaines et à la consommation alimentaire. Chez les poulettes Leghorn, le lot A est le plus retardé, il a pourtant ingéré plus d'énergie et de protéines que le lot $\mathrm{P}$ pour un développement pondéral semblable.

Nous avons enregistré une ponte satisfaisante à la suite de la restriction alimentaire des poulettes comme l'ont observé la plupart des auteurs. Nous montrons que leurs conclusions sont encore vraies même si un rationnement sévère est appliqué dès l'éclosion.

Nous pouvons donc affirmer que sur le plan nutritionnel, les performances de ponte ne dépendent que pour une faible part de la période de croissance. Cette dernière ne peut avoir une influence préjudiciable que pour un apport alimentaire très déficient (lots $\mathrm{P}$ et $\mathrm{A}$ ). Enn régime équilibré, une restriction quantitative modérée, telle que celle imposée au lot $S$ permet de réaliser une économie d'aliment sans jamais nuire à la ponte. Dans ce lot, on note seulement un retard de la maturité sexuelle; le poids moyen de l'œuf demeure égal à celui des témoins dès le début et tout au long de la ponte.

Dans les lots $\mathrm{P}$ et $\mathrm{A}$, au contraire, le poids de l'œuf est réduit; la comparaison des moyennes mensuelles montre un retard presque constant et significatif (fig. 2). A notre connaissance, une seule étude, celle de TrammEL, GREGER et Couch (I968) 
mentionne un effet semblable. Distribué de o à 6 semaines, un régime pauvre en lysine provoque une diminution du poids de l'œuf pendant la ponte ; distribué au-delà de 6 semaines, le même régime retarde la croissance sans avoir cet effet. Une déficience en acide aminé ou en protéines intervenant dans le jeune âge pourrait donc retarder l'augmentation du poids de l'œuf ; ce retard étant compensé par un accroissement du nombre de gros œufs pondus à la fin de la période de ponte. Il a été montré que le rationnement quantitatif de la poule pondeuse pouvait freiner lui aussi l'augmentation du poids de 1'œuf sans diminuer la production (SchumaIER et McGinnis, I968). L'observation des poules restreintes pendant leur jeune âge a montré qu'elles consommaient plus lentement leur ration. Autrement dit, le comportement alimentaire des adultes paraît modifié dans le sens d'un " autorationnement » du fait des conditions nutritionnelles défectueuses subies pendant les premières semaines de vie. C'est peut-être une des causes de la réduction du poids de l'œuf dans les lots $P$ et A dont l'alimentation doit satisfaire à la fois aux besoins de la ponte et à des besoins de croissance qui demeurent encore élevés.

\section{Différences entre souches.}

L'efficacité alimentaire des White Rock plus élevée pendant la croissance que celle des Leghorns concrétise l'effort de sélection qui a été faite dans ce sens pour les souches chair. Cette propriété se manifeste d'autant mieux que les conditions alimentaires sont sévères; elle demeure ensuite chez la pondeuse lorsque le rationnement est levé. Malgré 1'accroissement pondéral considérable de certains lots de White Rock adultes, la consommation de ces poules est parfaitement réglée. Par rapport aux témoins les ingérés sont plutôt moins élevés dans les lots antérieurement pénalisés bien que le poids total des œufs exportés soit significativement plus élevé. Ainsi l'alimentation ad libitum de ces animaux pendant le jeune âge se traduit par un double gaspillage : mauvaise utilisation azotée pendant la croissance, production inférieure pendant la ponte.

Chez les Leghorn, c'est exactement l'inverse : les lots très restreints pendant le jeune âge consomment plus et pondent moins. On peut se demander alors si les effets observés ne sont pas la conséquence d'un comportement acquis pendant le jeune âge et dont les effets ont des conséquences opposées selon les souches.

Le rationnement dans le jeune âge entraîne l'animal à limiter sa consommation même si les conditions pléthoriques surviennent. Dans ce cas, la consommation augmente mais pas suffisamment pour répondre à tous les besoins des animaux.

Chez les Leghorn, par exemple, souche maigre et légère un apprentissage trop sévère à la sobriété a vraisemblablement laissé les poules en mauvaise condition à l'entrée en ponte et ne leur a pas permis de surconsommer suffisamment pendant la ponte pour y remédier.

Chez les White Rock, au contraire, ce même entraînement a été bénéfique et a limité l'engraissement exagéré des sujets que l'on note à l'entrée et pendant la ponte.

Le seul comportement alimentaire n'explique cependant pas tout car l'on ne comprend pas bien pourquoi les restrictions protéiques sévères ont toujours un effet dépressif sur le poids moyen de l'œuf à un âge donné ; observation très rarement relatée dans la bibliographie. On peut penser que ce type de restriction trop précoce perturbe le développement de certains organes ou certains tissus de façon si profonde 
que l'organisme est incapable de compenser entièrement. Ainsi se confirmerait l'importance du jeune âge tant pour l'acquisition de comportements favorables que par la modification durable de certaines fonctions.

\section{CONCLUSION}

Sans pouvoir édicter des programmes nutritionnels définitifs dès cette première expérience, nous pouvons cependant dégager quelques conclusions pratiques et des perspectives nouvelles.

- Il est possible de débuter les restrictions alimentaires dès l'éclosion sans diminuer les performances de la ponte. La distribution d'un régime complet en quantité limitée aboutit à coup sûr à des résultats satisfaisants quelle que soit la souche. Mais la distribution précoce d'un régime déficient en protéines ou en acides aminés n'est pas recommandable pour les souches de type Leghorn.

- Dans le cas des souches lourdes destinées à la production du poulet de "chair", toutes les méthodes de restriction paraissent valables; le choix de l'une d'entre elles dépend donc essentiellement de critères économiques. Les restrictions subies pendant les premières semaines de vie semblent influencer favorablement le comportement alimentaire des adultes en empêchant la surconsommation en cours de ponte. De toute façon, l'habitude qui prévaut, de suralimenter les poussins pour restreindre ensuite les poulettes considérablement, paraît tout à fait irrationnelle. Le meilleur résultat devrait être obtenu avec une croissance ralentie, mais progressive de manière à éviter tout " stress ".

- Partant de ces considérations, on peut envisager de nouveaux plans de rationnement pour les futures reproductrices de souche lourde. L'utilisation d'un régime unique pendant toute la période d'élevage paraît possible. Ce régime du type de ceux habituellement distribués en fin de croissance serait fourni ad libitum pendant les premières semaines de vie; ce rationnement qualitatif devrait être suivi par un rationnement quantitatif pour que le taux de croissance demeure à peu près constant jusqu'à l'âge d'entrée en ponte. En ce qui concerne l'alimentation alternée, les résultats obtenus rendent plausible l'utilisation par périodes successives d'un aliment complet et de céréales produites sur place en cherchant à appliquer comme précédemment une déficience qualitative en début de croissance et un rationnement quantitatif ensuite.

$$
\text { Reçu pour publication en août } 1970 .
$$

\section{SUMMARY}

EFFECT OF RESTRICTED FEEDING SINCE BIRTH UPON THE LAYING PERFORMANCES OF TWO BREEDS OF PULLETS

I 200 pullets from each breed, the one being heavy (White Rock type) and the other light (Leghorn type) were distributed into 4 groups after hatching. The control groups were fed a balanced diet all the time allowing rapid growth. The $S$ groups received the same diets, but were fed according to the skip-a-day system : 6 days out of 7 until 12 weeks of age, 5 days out of 
7 between $\mathrm{I} 2$ and 20 weeks. Until the age of 3 weeks, cereals were used as protein source for the P groups (Io p. IOO), the protein level being afterwards raised to I 3 p. Ioo by means of animal meals. The A groups received alternatively ro p. roo protein (the cereal diet) during I 4 days and $2 \mathrm{I}$ p. Ioo protein during 7 days. This rythm (I4 days-7 days) was maintained during the whole growing period. The rations were supplied ad libitum to all the animals.

From 20 weeks of age and during the whole laying period, all the animals from the same breed received an identical diet ad libitum satisfying their requirements. The influence of restricted feeding during the young age was particularly pronounced in the groups $P$ and $A$ : important slackening of growth, delay in sexual maturity of about 20 days. There was a very significant interaction between the breed and type of restriction. In the Leghorn pullets, the greatest delay in sexual maturity was observed in group $\mathrm{A}$; in the White Rock pullets in group P.

As far as these two breeds are concerned, the laying performances are not greatly changed by the food restrictions imposed during the young age. However, the egg production of the Leghorn was slightly, but significantly reduced in the most restricted groups (P and $\mathrm{A}$ ). On the contrary, in the White Rock breed, food restrictions always have a favourable effect upon their further performances. In these groups, the mean weight of the egg at a given age is always reduced.

\section{RÉFÉRENCES BIBLIOGRAPHIQUES}

Hutt F. B., Nesheim M. C., I 969 . Genetic differences in reproduction after early retardation of growth on a deficient diet. Poult. Sci., 48, 1825.

Schumaier G. W., McGinnis J., 1968. Limited time feeding of heavy bred pullets. Poult. Sci., 47, I7 16.

Simon J., Blum J. C., Jacouot R, rg68. Étude des effets de l'alimentation discontinue chez le poulet : variation pondérale et composition corporelle. C. R. Acad. Sci. Paris, 267, 2002-2004

Singsen E. P., Nagel J., Patrick S. G., Matterson L. D., rg65. The effect of a lysine deficiency on growth characteristics, age at sexual maturity and reproductive performance of meat type pullets. Poult. Sci., 44, 1467-I473.

Snedecor G. W., r956. Statistics methods. The Iowa State College Press, Ames (Iowa).

TRAMmel J. L., CReger C. R., Couch J. R., xg68. Low lysine diets for rearing replacement pullets. Poult. Sci., 47, 1727. 